\title{
A 275-425-GHz Tunerless Waveguide Receiver Based on AlN-Barrier SIS Technology
}

Jacob W. Kooi, Attila Kovács, Matthew. C. Sumner, Goutam Chattopadhyay, Senior Member, IEEE, Riley Ceria, Dave Miller, Bruce Bumble, Henry G. LeDuc, Jeffrey A. Stern, and Tom G. Phillips

\begin{abstract}
We report on a 275-425-GHz tunerless waveguide receiver with a 3.5-8-GHz IF. As the mixing element, we employ a high-current-density $\mathrm{Nb}-\mathrm{AIN}-\mathrm{Nb}$ superconducting-insulatingsuperconducting (SIS) tunnel junction. Thanks to the combined use of AIN-barrier SIS technology and a broad bandwidth waveguide to thin-film microstrip transition, we are able to achieve an unprecedented $43 \%$ instantaneous bandwidth, limited by the receiver's corrugated feedhorn.

The measured double-sideband (DSB) receiver noise temperature, uncorrected for optics loss, ranges from $55 \mathrm{~K}$ at $275 \mathrm{GHz}$, $48 \mathrm{~K}$ at $345 \mathrm{GHz}$, to $72 \mathrm{~K}$ at $425 \mathrm{GHz}$. In this frequency range, the mixer has a DSB conversion loss of $2.3 \pm 1 \mathrm{~dB}$. The intrinsic mixer noise is found to vary between $17-19 \mathrm{~K}$, of which $9 \mathrm{~K}$ is attributed to shot noise associated with leakage current below the gap. To improve reliability, the IF circuit and bias injection are entirely planar by design. The instrument was successfully installed at the Caltech Submillimeter Observatory (CSO), Mauna Kea, HI, in October 2006.
\end{abstract}

Index Terms-Allan variance, AIN tunnel barrier, broadband waveguide transition, dc break, heterodyne receiver, high current density, IF match, multiple Andreev reflection (MAR), planar bandpass filter, shot noise, superconducting-insulator-superconducting (SIS) mixer, system stability.

\section{INTRODUCTION}

A LL THE pre-existing superconducting-insulator-superconducting (SIS) waveguide receivers at the Caltech Submillimeter Observatory (CSO), Mauna Kea, HI, use waveguide tuners to achieve sensitivities a few times the quantum noise limit. Each of these receivers has played a pioneering role in the submillimeter field. However, modern astronomy is demanding more capability in terms of sensitivity, bandwidth, stability, frequency agility, and ease of use. To facilitate these requirements, technological advances of the past decade have enabled receiver designers to construct tunerless receivers with expanded IF bandwidths at sensitivities a few times the

\footnotetext{
Manuscript received April 9, 2007; revised July 11, 2007. This work was supported in part by the National Science Foundation under Grant AST-0540882.

J. W. Kooi, M. C. Sumner, R. Ceria, D. Miller, and T. G. Phillips are with the Submillimeter Astronomy and Instrumentation Group, California Institute of Technology, Pasadena, CA 91125 USA (e-mail: kooi@ submm.caltech.edu; sumner@submm.caltech.edu; davem@submm.caltech.edu; tgp@submm. caltech.edu).

A. Kovács is with the Max Planck Institute für Radio Astronomy, 53121 Bonn, Germany (e-mail: attila@ submm.caltech.edu).

G. Chattopadhyay, B. Bumble, H. G. LeDuc, and J. A. Stern are with the Jet Propulsion Laboratory, Pasadena, CA 91109 USA (e-mail: goutam@submm. caltech.edu; Bruce.Bumble@jpl.nasa.gov; leduc@jpl.nasa.gov; Jeffrey.Stern@ jpl.nasa.gov).

Digital Object Identifier 10.1109/TMTT.2007.905503
}

quantum noise limit. Although different in detail and configuration, advanced receiver designs now feature prominently in, for example, the Heterodyne Instrument for the Far-Infrared (HIFI) on the Herschel satellite, ${ }^{1} \mathrm{ALMA},{ }^{2}$ the Plateau de Bure interferometer (IRAM), ${ }^{3}$ the Atacama pathfinder experiment (APEX), ${ }^{4}$ TELIS, ${ }^{5}$ and the Harvard-Smithsonian Submillimeter Array (SMA). 6

To upgrade the heterodyne facility instrumentation at the CSO, four tunerless balanced-input waveguide receivers have been designed to cover the $180-720-\mathrm{GHz}$ frequency range [1], [2]. These receivers will allow dual-frequency (two-color) observations in the 230/460- and 345/660-GHz atmospheric windows. The IF bandwidth of the CSO receivers will increase from the current 1 to $4 \mathrm{GHz}$, though in principle, $12 \mathrm{GHz}$ is possible. For spectroscopic studies of distant galaxies, a complementary two-channel $275-425-\mathrm{GHz}$ balanced continuouscomparison (correlation) receiver [3] is also under construction. Balanced configurations were chosen for their inherent local oscillator (LO) amplitude noise cancellation properties, facilitating the use of synthesizer-driven LO chains. Unique to the CSO, broad RF bandwidth is favored, allowing the same science to be done with fewer instruments. To maximize the RF bandwidth, we explore the use of high current-density AlN-barrier SIS technology in combination with a broad bandwidth full-height waveguide to thin-film microstrip transition. Additional advantages of AlN tunnel barriers, compared to $\mathrm{AlO}_{x}$-barriers, are enhanced chemical robustness and a low $\omega R C$ product (increased RF bandwidth). Even if optimal RF bandwidth is not a requirement, a low $\omega R C$ product provides a more homogeneous frequency response and increased tolerance to errors in device fabrication.

To validate the many technologies used in the design process, we have constructed a technology demonstration receiver ("Trex") to cover the important $275-425-\mathrm{GHz}$ atmospheric windows. The design principles laid out in this paper are directly applicable to the entire set of new CSO receivers. The receiver offers a $43 \% \mathrm{RF}$ bandwidth, nearly $50 \%$ wider than the ALMA bands 7 275-373-GHz specification [4], and limited to a large extent by the corrugated feedhorn characteristics.

\footnotetext{
${ }^{1}$ [Online]. Available: http://www.sron.nl/divisions/lea/hifi/

${ }^{2}$ [Online]. Available: http://www.alma.info/

${ }^{3}$ [Online]. Available: http://iram.fr/

${ }^{4}$ [Online]. Available: http://www.apex-telescope.org/

5 [Online]. Available: http://www.sron.nl/

${ }^{6}$ [Online]. Available: http://sma-www.harvard.edu/
} 


\section{INSTRUMENT DESIGN}

\section{A. Broad Bandwidth Waveguide to Microstrip Transition}

Traditionally the majority of SIS and hot electron bolometer (HEB) waveguide mixers employ planar probes that extend all the way across the waveguide [5]-[9]. An important reason for the popularity of this design is the convenience with which the active device can be biased and the IF signal extracted. Unfortunately, the "double-sided" (balanced) probe exhibits a rather poor RF bandwidth $(\leq 15 \%)$, when constructed in a full-height waveguide. When the height of the waveguide is reduced by $50 \%$, the probe's fractional bandwidth improves dramatically to a maximum of approximately $33 \%$. These results can be understood in that the popular double-sided probe is essentially a planar variation of the well known Eisenhart and Khan waveguide probe [10]. Borrowing from Withington and Yassin's assessment [11], the real part of the probe's input impedance is influenced in a complex way by the parallel sum of individual nonpropagating modal impedances, and as such, is frequency dependent. By lowering the height of the waveguide, the effect of nonpropagating modes may be reduced [12]-[15].

An alternative approach is to use an asymmetric probe that does not extend all the way across the waveguide. For this type of probe, the modal impedances add in series. The real part of the input impedance depends only on the single propagating mode and is relatively frequency independent. These probes are typically implemented in full-height waveguide, which minimizes conduction loss and reduces the complexity of fabrication. A rectangular version of the "one-sided" probe has been used quite extensively by microwave engineers [16], [17], was introduced to the submillimeter community by Kerr and Pan [18] in 1990, and is currently part of the baseline design for ALMA band 3 and 6 [19], [20]. The radial probe described here represents an attempt to extend the use of radial modes to the waveguide coupling problem [21]. From a practical point of view, the radial probe can be quite naturally made to feed a thin-film microstrip or coplanar wave (CPW) line that has a small linewidth and thin insulator [22], [23].

If very broadband operation is desired, a further improvement in the probe's performance can be achieved by adding a simple capacitive waveguide tuning step in front of the probe (Fig. 1). Typically, a $15 \%$ reduction in waveguide height is adequate to tune out most of the probe's residual reactance. The length of the step is on the order of the waveguide height dimension. Since some of the reactance in the probe is tuned out by the step, the distance between the substrate and backshort must be increased by $1.5 \%-2.0 \% \lambda_{g}$. This increase in distance represents an added advantage to using a waveguide step, as it reduces machining difficulty. Fig. 2 depicts the radial probe input return loss and impedance locus.

To simplify the way the IF signal is extracted, it is possible to go across the waveguide with an inductive quarter-wavelength meandering transmission line [24]-[26]. In this case, care must be taken not to excite modes that result in high- $Q$ resonances in the probe's passband. Through extensive simulations [29], we find that going across the waveguide in this manner reduces symmetrically the transition bandwidth by $\sim 7 \%$, which is likely to be acceptable for many applications. To match the very

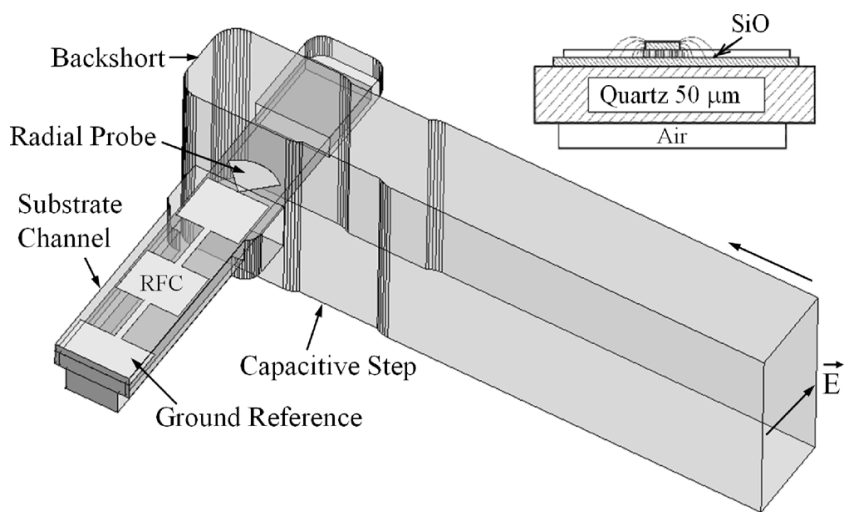

Fig. 1. Isometric view of the waveguide transition. The $90^{\circ}$ radial probe couples very efficiently to the fundamental $\mathrm{TE}_{10}$ waveguide mode. It is important to have the probe situated on a suspended dielectric, as this maximizes the width of the substrate and makes room for the radial fan. Widening of the substrate channel around the first section of the RF choke serves to reduce the imaginary component of the probe impedance. To help reduce the probe's input return loss and increase the RF bandwidth even further, the addition of a small capacitive waveguide tuning step in front of the radial probe is found useful. Dimensions are provided in Table I.

TABLE I

PARAMETERS USED FOR THE 270-430-GHz DESIGN

\begin{tabular}{ll}
\hline \hline Substrate material & quartz \\
Waveguide size $(\mu \mathrm{m})$ & $580 \times 270$ \\
Probe radius $(\mu \mathrm{m})$ & 112 \\
Substrate width $(\mu \mathrm{m})$ & 200 \\
Substrate thickness $(\mu \mathrm{m})$ & 50 \\
Air height above substrate $(\mu \mathrm{m})$ & 25 \\
Air height below substrate $(\mu \mathrm{m})$ & 50 \\
Backshort-substrate distance $(\mu \mathrm{m})$ & 103 \\
Backshort radius $(\mu \mathrm{m})$ & 70 \\
Probe impedance $(\Omega)$ & $47+\mathrm{i} 3$ \\
\hline \hline
\end{tabular}

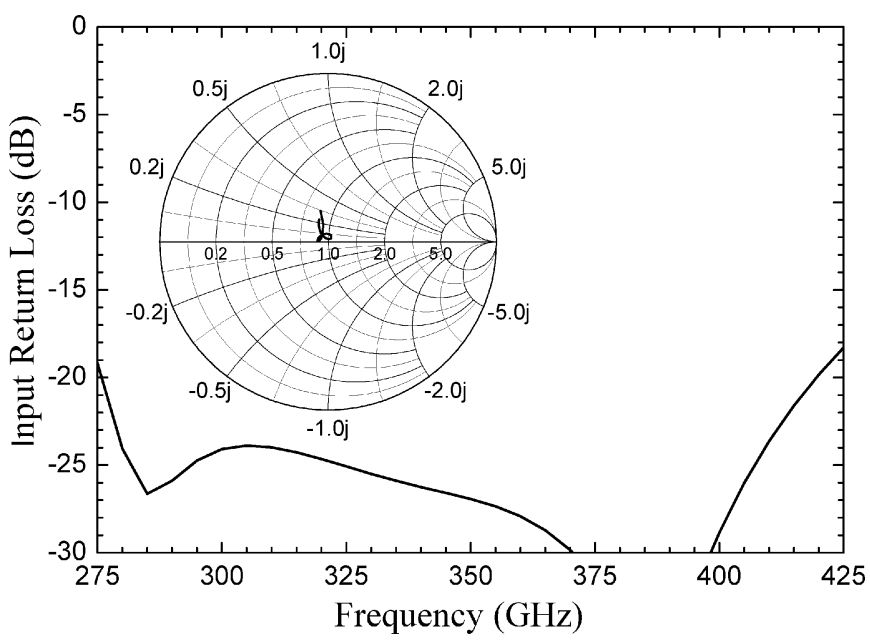

Fig. 2. Input return loss of the fixed tuned full-height waveguide transition The fractional bandwidth of the $350-\mathrm{GHz}$ full-height waveguide radial probe is $\approx 45 \%, 95 \%$ of a standard $\mathrm{TE}_{10}$ fundamental waveguide band. The impedance locus of the probe is $47+i 3 \Omega$.

large instantaneous RF bandwidth afforded by an AlN-barrier twin-junction SIS matching circuit, we have opted in our design for the $100 \%$ asymmetric radial probe. 


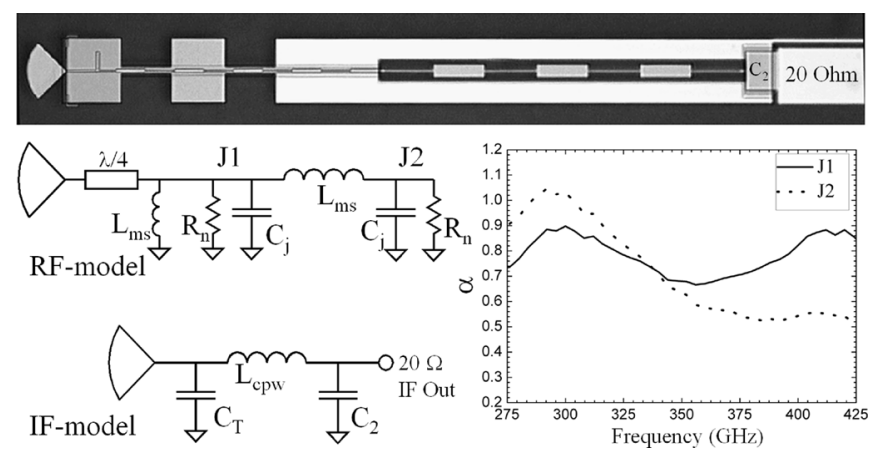

Fig. 3. 350-GHz junction layout and simulated LO pumping parameter $\alpha$. The radial probe antenna is visible on the left side. The IF signal is taken out via a microstrip RF choke (on 300-nm SiO, $\epsilon_{r}=5.6$ ) that connects to a high-impedance CPW transmission line (inductive) and integrated shunt capacitor $\left(C_{2}\right)$. This $L-C$ mechanism provides a $\pi$ tuning network with the combined capacitance of the probe, twin-junction RF tuning structure, and microstrip RF matching network $\left(C_{T}\right)$. The passband is optimized to cover $1-13 \mathrm{GHz}$. To minimize gain compression, the integrated shunt capacitor also serves to terminate out-of-band broadband noise. The IF impedance presented to the twin SIS junctions is $14 \Omega$, $\sim 2.7 R_{n}$

\section{B. New Set of SIS Junctions}

Circuit design of the high current-density niobium SIS junctions (four frequency bands) was done at the California Institute of Technology, Pasadena, with fabrication at the Jet Propulsion Laboratory (JPL). The new SIS tunnel junctions all share the same $50-\mu \mathrm{m}$-thick quartz wafer. The design employs twin SIS junctions with AlN barriers and a current density of $25 \mathrm{kA} / \mathrm{cm}^{2}$. In our design, we have used Supermix [27], [28], a flexible software library for high-frequency harmonic-balanced mixer and superconducting circuit simulation, in combination with extensive 2-D and 3-D electromagnetic (EM)-field analysis of the RF and IF embedding circuitry [29], [30]. Based on extensive simulation, the twin-junction RF matching network was found to exhibit a slightly larger RF bandwidth then the more common single-junction RF matching network [31], [32]. The AlN-barrier SIS junction $\left[R_{n} C\right]^{-1}$ product is $164 \mathrm{GHz}$, similar to the bandwidth afforded by the thin-film waveguide transition, and high enough to avoid the Body-Fano bandwidth limitation. As part of the AlN-barrier characterization process at the JPL, the specific junction capacitance is estimated at $80 \mathrm{fF} / \mu \mathrm{m}^{2}$. To minimize saturation $\left(\delta V_{\text {sis }} \propto\left[P_{\text {sig }} R_{n}\right]^{0.5}\right)$, while maintaining reasonably sized junction areas, we decided on a 5.4- $\Omega$ twin junction normal state resistance. The calculated bias voltage variation $\left(\delta V_{\text {sis }}\right)$ between 0 - and $300-\mathrm{K}$ loads is $80 \mu \mathrm{V} \mathrm{rms}$, assuming a $160-\mathrm{GHz} \mathrm{RF}$ noise bandwidth. From the curvature of the measured total-power response, we estimate the saturation $\leq 1 \%$.

Matching to an IF impedance of $20 \Omega$ is realized on-chip (Fig. 3). The choice of this impedance is dictated by the limited available real estate, and the need to minimize gain compression [33]. To transform the $20-\Omega$ mixer-chip IF output impedance to a $50-\Omega$ load, an external matching network/bias tee is employed (Section II-E). The mixer design has been optimized for minimum noise temperature and optimal conversion gain from 275 to $425 \mathrm{GHz}$, while simultaneously regulating the RF and IF input return loss to $\geq 8 \mathrm{~dB}$. The latter is important, as reflections from the RF or IF port can lead to mixer instability

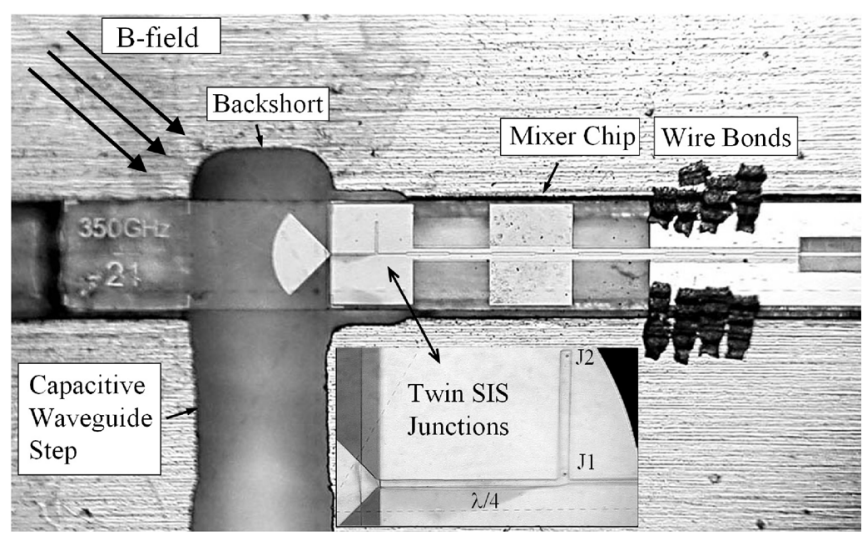

Fig. 4. 350-GHz mixer chip mounted in the IF channel with the radial probe extruding into the waveguide. Note the capacitive waveguide step directly in front of the probe. Wire bonds are used to provide the ground reference.

TABLE II

DEVICE PARAMETERS

\begin{tabular}{llll}
\hline \hline Parameter & B030925 & B030926 & Specification \\
\hline $\mathrm{R}_{n}(\Omega)$ & 11.8 & 10.9 & $10.9 \pm 15 \%$ \\
$\mathrm{C}_{s}\left(\mathrm{fF} / \mu \mathrm{m}^{2}\right)$ & 80 & 80 & $\pm 10 \%$ \\
$\mathrm{Area}\left(\mu \mathrm{m}^{2}\right)$ & 0.7 & 0.7 & $1 \times 0.7 \pm 15 \%$ \\
$\mathrm{R}_{n} \mathrm{~A}\left(\Omega \mu \mathrm{m}^{2}\right)$ & 8.28 & 7.63 & $7.6 \pm 15 \%$ \\
$\mathrm{R}_{s g} / \mathrm{R}_{n}$ & $9-11$ & $10-12$ & 15 \\
Vgap $(\mathrm{mV})$ & $2.55-2.60$ & $2.68-2.75$ & 2.90 \\
$\mathrm{Nb} T o p(\mathrm{~nm})$ & 420 & 420 & $400 \pm 20 \%$ \\
$\mathrm{SiO}(\mathrm{nm})$ & 320 & 320 & $300 \pm 15 \%$ \\
$\mathrm{Nb}$ Bottom $(\mathrm{nm})$ & 210 & 210 & $200 \pm 20 \%$ \\
\hline \hline
\end{tabular}

(Section III-D). In Fig. 4, we show a photograph of the mixer chip positioned in the waveguide. Short parallel wire bonds provide the ground contact.

The tunnel junction under discussion is from batch B030926. It has a measured $R_{n} A$ product of $7.6 \Omega \mu \mathrm{m}^{2}$, a junction area of $1 \times 0.7 \mu \mathrm{m}^{2}, R_{\mathrm{sg}} / R_{n}$ ratio of 13.8 at $2.0-\mathrm{mV}$ bias, and a 5.32- $\Omega$ normal-state resistance. At the CSO, on top of Mauna Kea, HI, a lower LHe bath temperature $(3.67 \mathrm{~K})$ results in a subgap leakage current reduction of $10 \%\left(R_{\mathrm{sg}} / R_{n}=15\right)$. For the two successful batches produced by the JPL, the specifications are shown in Table II.

\section{Nb/Aln ${ }_{x}-A l / N b$ Junction Fabrication}

Devices are fabricated on $z$-cut crystalline-quartz wafers $250-\mu \mathrm{m}$ thick, $76 \mathrm{~mm}$ in diameter, and polished on both sides. Magnetron sputter deposition and room-temperature nitride growth is done in-situ. For this we use a load-locked ultrahigh vacuum system with a typical base pressure of $2 \times 10^{-7} \mathrm{~Pa}$. The trilayer is deposited by a liftoff process employing a multilayer photolithographic technique using PMMA under AZ5214 photoresist. Optical lithography is accomplished by means of a GCA model 6300 i-line $(365 \mathrm{~nm})$ wafer stepper/aligner tool. The resulting undercut structure allows for clean liftoff of the sputtered films. This step forms the ground-plane structure with layers of $180-\mathrm{nm} \mathrm{Nb}$ base, 6-nm Al, $\mathrm{AlN}_{x}$, and 80-nm $\mathrm{Nb}$ counter electrode. $\mathrm{AlN}_{x}$ barrier formation is done with a pure $\mathrm{N}_{2}$ low-pressure plasma. The substrate is held at ground potential and an opposing electrode is driven by a $13.5-\mathrm{MHz}$ source to create the $\mathrm{N}_{2}$ plasma. A rectangular junction mesa, 
the smallest being $1.0 \mu \mathrm{m} \times 0.5 \mu \mathrm{m}$ in area, is defined by direct-write electron-beam lithography in a 100-nm-thick PMMA stencil. Chromium is deposited through the PMMA stencil and serves as an etch mask over $500 \mathrm{~nm}$ of polyamide. Contact regions of the trilayer are then protected by adding a photoresist stencil. The combined chromium + photoresist/polyamide structure is etched using an oxygen reactive ion etch (RIE). The polyamide remaining defines an isolation window and junction mesa for subsequent $\mathrm{Nb}$ RIE. To achieve $\mathrm{Nb}$ etch directionality, we utilize a gas mixture of $62 \% \mathrm{CCl}_{2} \mathrm{~F}_{2}+31 \% \mathrm{CF}_{4}+7 \% \mathrm{O}_{2}$.

Electrical isolation of the base electrode from the microstrip wire layer is provided by thermal evaporation of $300 \mathrm{~nm}$ of $\mathrm{SiO}$. Samples are rotated at a slight tilt angle during $\mathrm{SiO}$ deposition to assure good isolation and self-aligned liftoff with the polyamide. Liftoff is then accomplished by dissolving the polyamide in a resist stripper containing NMP.

The waveguide probe and wire layer is formed by a blanket deposition of 400-nm Nb. RIE etching with an OiR620 photoresist stencil defines this pattern. Gold contacts are patterned by liftoff of films done by evaporation. As a final step, the substrates are diced into near $\mathrm{cm}^{2}$ size chips and diagnostic sites are tested. The thick chips were lapped by Ron Kehl Engineering, ${ }^{7}$ down to $50-\mu \mathrm{m}$ thickness, with the individual mixer elements diced out at the JPL using a Disco saw and diamond/Ni blade.

\section{Multiple Andreev Reflection (MAR)}

It is found, not surprisingly, that high current-density $\left(J_{c}\right)$ AlN-barrier tunnel junctions exhibit a larger leakage current than commonly used lower $J_{c} \mathrm{AlO}_{x}$ tunnel junctions $\left(R_{\mathrm{sg}} / R_{n}\right.$ ratios of 10-14 versus 20-35). To investigate the contribution of the higher subgap leakage to the intrinsic mixer noise, we apply a technique described by Dieleman et al. [34]. In that analysis, the noise spectral density below the energy gap is modeled by summing the thermalized single-electron tunnel current $\left(I_{\text {tun }}\right)$ with a charged quantum transport current $\left(I_{\mathrm{mar}}\right)$ that results from MARs through pinholes in the barrier. We find that

$$
S_{I}(V)=2 e I_{\text {tun }}+2 q(V) I_{\text {mar }}, \quad q(V)=(1+2 \Delta / e V)
$$

with $2 \Delta / e=2.75 \mathrm{mV}$ for our AlN junctions. Rearranging (1) by defining $r=I_{\text {tun }} / I$ with $I=\left(I_{\text {tun }}+I_{\text {mar }}\right)$ gives

$$
S_{I}(V)=2 e I\left[1+\frac{2 \Delta}{e V}(1-r)\right]
$$

The noise contribution of the junction to the IF output is then given by

$$
P_{\mathrm{IF}}=G_{\mathrm{IF}} B\left[\frac{S_{I}(V) R_{d}}{4}\left(1-\Gamma_{\mathrm{IF}}^{2}\right)\right]
$$

where

$$
\Gamma_{\mathrm{IF}}=\frac{R_{d}-Z_{o}}{R_{d}+Z_{o}}
$$

${ }^{7}$ Ron Kehl Engineering, San Jose, CA.

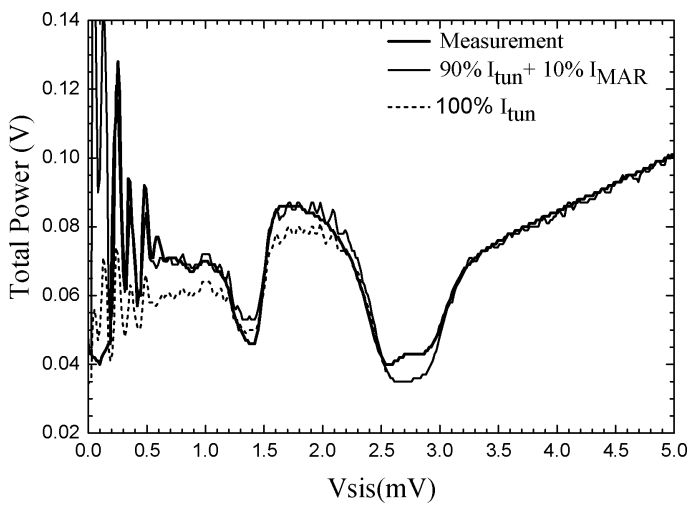

Fig. 5. Measured mixer output noise compared to single-electron and MARenhanced shot noise. A 10\% MAR current accounts for all of the excess subgap noise, indicative that nearly all of the shot noise is due to single-electron transport in the barrier. The total intrinsic shot + MAR noise in the junction at 2-mV bias is $8.9 \mathrm{~K}, \sim 45 \%$ of the derived mixer noise (Section III-B).

$G_{\mathrm{IF}}$ is the IF gain, $B$ is the IF bandwidth, $R_{d}$ is the differential resistance obtained from the measured unpumped $I / V$ curve, and $Z_{o}$ is the IF impedance. To properly account for all the subgap mixer output noise, we need a $10 \%$ MAR current (Fig. 5). When compared to an idealized junction with $R_{\mathrm{sg}} / R_{n}=30$, we conclude that $90 \%$ of the enhanced subgap noise is due to single-electron tunneling. From this the net increase in mixer noise is estimated to be $8.9 \mathrm{~K}$.

In Section III-B, we calculate the IF noise contribution by biasing the junction above the gap. In this case, the current will be entirely due to single-electron tunneling. $T_{\text {shot }}$ may then be found in the traditional way

$$
T_{\text {shot }}=\frac{e R_{d} I}{2 K_{b}} \operatorname{coth}\left(\frac{e V}{2 K_{b} T}\right) \text {. }
$$

\section{E. Planar 4-8-GHz IF Matching Network, DC Break, Bias Tee, and Electromagnetic Interference (EMI) Filter}

In a practical mixer configuration, the active device is terminated into a desired IF load impedance, the bias lines filtered and injected via a bias tee, and the output dc decoupled. The dc block is usually accomplished with a small surface-mount capacitor contacted in series by either a soldered contact or wire bond. As a consequence, parasitic resonances at the upper end of the IF band are easily excited. Moreover, since the dc-blocking capacitor passes the mixer IF output current, a failure would be catastrophic. Indeed, component failure can occur in may ways, the most obvious perhaps due to mechanical stress from repeated cryogenic thermal cycles. As an alternative, we investigate the use of parallel-coupled suspended microstrip lines [35]-[37]. An important advantage of this planar approach is that it affords accurate modeling with 3-D EM field-simulation software [29].

As shown in Fig. 6, the suspended coupled microstrip lines act as a compact bandpass filter. For this filter to work, the ground plane directly underneath the filter needs to be removed. The IF board is optically positioned on top of a machined cutout (resonant cavity) by a set of alignment holes. The advantages of this technique are simplicity of design (only one lithography 


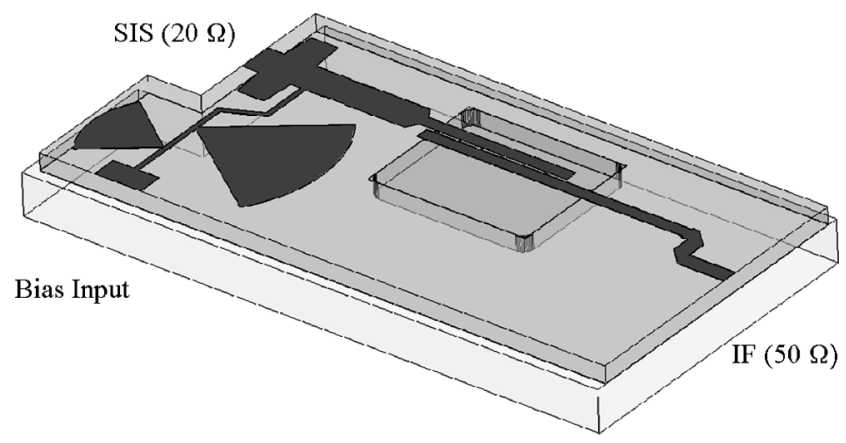

Fig. 6. IF configuration with a 3-9-GHz passband response. The combined IF match, dc-break, bias tee, and EMI filter are planar by design. Design parameters are provided in Table III.

TABLE III

COUPLING PARAMETERS OF FIG. 6

\begin{tabular}{ll}
\hline \hline Substrate & Alumina \\
$\boldsymbol{\epsilon}_{r}$ & 9.80 \\
$\mathrm{H}_{\text {sub }}(\mu \mathrm{m})$ & 635 \\
$\mathrm{~W}(\mu \mathrm{m})$ & 480 \\
$\mathrm{~L}(\mathrm{~mm})$ & 5.72 \\
$\mathrm{~S}(\mu \mathrm{m})$ & 120 \\
$\mathrm{H}_{\text {cav }}(\mu \mathrm{m})$ & 585 \\
$\mathrm{H}_{\text {air }}(\mathrm{mm})$ & 2.5 \\
$\mathrm{~L}_{c} \times \mathrm{W}_{c}(\mathrm{~mm})$ & $5.08 \times 6.1$ \\
\hline \hline
\end{tabular}

$H_{\text {sub }}$ denotes the substrate height, $W$ the width of the coupled lines, $L$ their length, $S$ the spacing, $H_{\text {cav }}$ the cavity depth, $H_{\text {air }}$ the air height above the substrate, $L_{c}$ the cavity length, and $W_{c}$ the cavity width. Center frequency is $6 \mathrm{GHz}$.

step) and improved reliability. The disadvantage at lower IF frequencies is size, $\lambda_{g} / 4$. To secure the alumina board ${ }^{8}$ to the $\mathrm{Au}$ plated brass mixer block, we use an indium alloy paste. ${ }^{9}$ Repeated thermal cycles have proven successful, an indication that the thermal expansion of the dissimilar materials does not pose a significant problem.

Details of the blocking filter are summarized in Table III. The spacing $S$, and cavity depth $H_{\text {cav }}$ set the coupling. Tolerance values should be held to $\pm 5 \%$. Performance curves for IF coupling efficiency and impedance are shown in Fig. 7. In principle, the twin-junction SIS design affords a 1-13-GHz IF passband response. However, due to availability of low noise cryogenic components, we have chosen a design to match the $4-8-\mathrm{GHz}$ low-noise InP amplifier from Chalmers University, Gotëborg, Sweden (Section II-F) and the 4-8-GHz cryogenic isolator. ${ }^{10}$

\section{F. 4-8-GHz Low-Noise Cryogenic Amplifier}

In collaboration with Chalmers University and the National Aeronautics and Space Administration (NASA)'s JPL, we have acquired extremely low noise $(2.2 \mathrm{~K}) 4-8-\mathrm{GHz}$ indium phosphide (InP) cryogenic HEMT amplifiers [38], [39]. The dc power consumption is $\approx 10 \mathrm{~mW}$, allowing the amplifier to be mounted on the LHe stage, and the input return loss $18 \mathrm{~dB}$. The amplifier gain is $25 \mathrm{~dB}$ (two stages). To add additional gain at the 4-K stage, we use a cryogenic GaAs monolithic microwave

\footnotetext{
${ }^{8}$ American Technical Ceramics, Huntington Station, NY.

${ }^{9}$ Indalloy Paste, \#290, Indium Corporation of America, Singapore. [Online]. Available: www.indium.com

${ }^{10}$ Pamtech, Camarillo, CA.
}

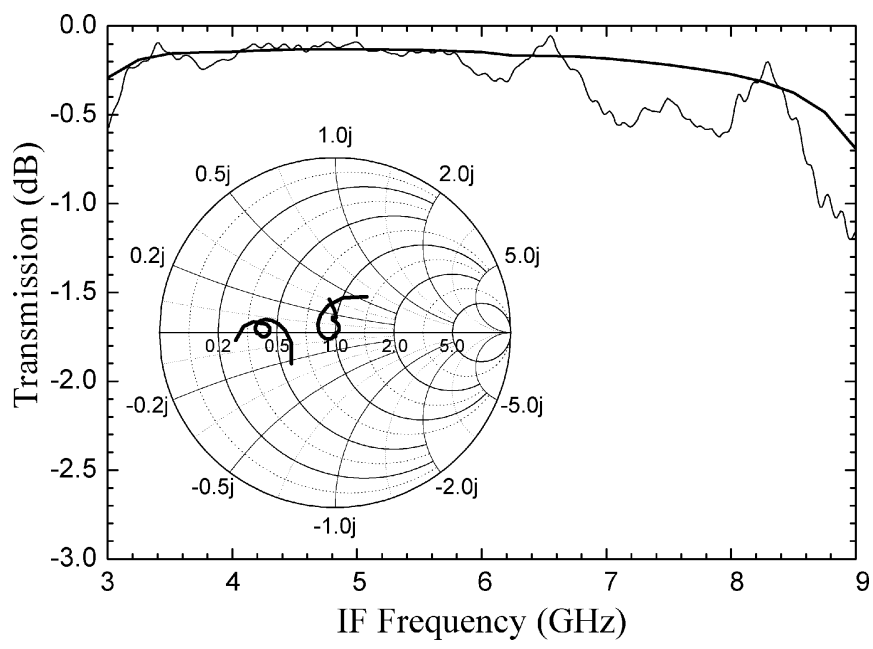

Fig. 7. Simulated and measured coupling efficiency into a $50-\Omega$ load (see also Fig. 6). Inset: simulated complex impedance towards the SIS chip $(20 \Omega)$ and IF output $(50 \Omega)$.

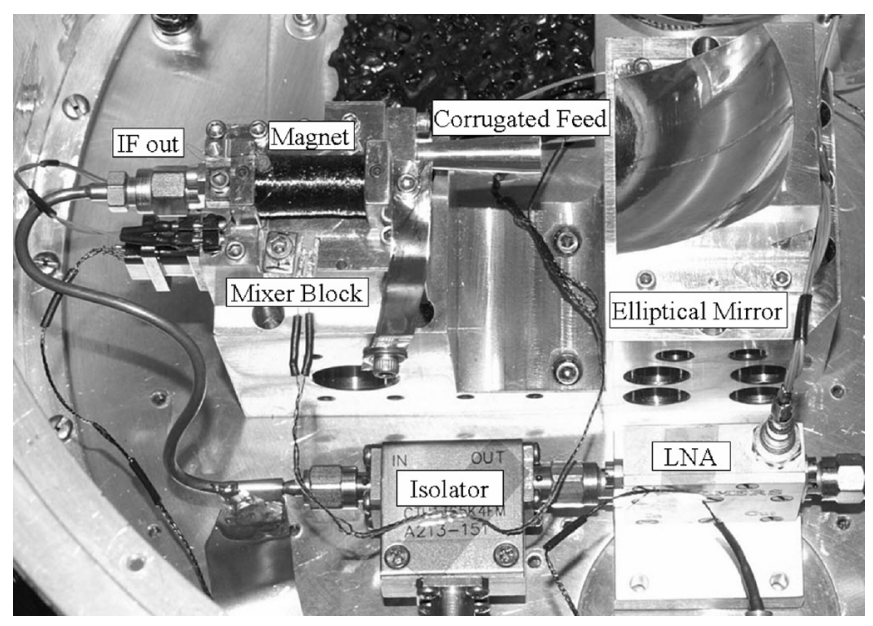

Fig. 8. Mixer, off-axis elliptical mirror, $4-8-\mathrm{GHz}$ isolator, and LNA are all situated on the LHe stage. The IR Laboratory's Dewar is mounted upside down in the Cassegrain focus of CSO.

integrated circuit (MMIC) from Prof. Weinreb's group at the California Institute of Technology [40] as a second low-noise amplifier (LNA). This amplifier offers excellent performance, with a gain of $25 \mathrm{~dB}$ and a noise temperature of $5 \mathrm{~K}$.

\section{G. Cooled Optics}

The receiver noise temperature is critically dependent on optical loss in front of the mixer. This can be understood from

$$
T_{\mathrm{rec}}^{\mathrm{DSB}}=T_{\mathrm{rf}}+\frac{T_{\mathrm{mix}}}{G_{\mathrm{rf}}}+\frac{T_{\mathrm{IF}}}{G_{\mathrm{rf}} G_{\mathrm{mix}}^{\mathrm{DSB}}} .
$$

$G_{\mathrm{mix}}^{\mathrm{DSB}}$ is the double-sideband (DSB) mixer gain, $G_{\mathrm{rf}}$ the front-end optics transmission coefficient, $T_{\mathrm{rf}}$ the optics noise temperature, $T_{\mathrm{IF}}$ is the IF noise temperature, and $T_{\text {mix }}$ the intrinsic mixer noise. We have minimized the optics noise by careful selection of the infrared (IR) blocking filters and vacuum window, and by using a cooled off-axis elliptical mirror. Fig. 8 depicts some of the receiver hardware mounted on the cryostat 
TABLE IV

MEASUREd AND CALCULATED RECEIVER PARAMETERS. REFER TO (6) FOR DETAILS. ${ }^{*} T_{\text {mix }}$ INCLUDES $9 \mathrm{~K}$ OF MAR-ENHANCED SHOT NOISE

\begin{tabular}{llll}
\hline \hline Parameters & $\mathbf{2 8 0} \mathbf{~ G H z}$ & $\mathbf{3 4 5} \mathbf{~ G H z}$ & $\mathbf{4 2 4} \mathbf{~ G H z}$ \\
\hline $\mathrm{T}_{r e c}^{D S B}(\mathrm{~K})$ & 50 & 48 & 72 \\
$\mathrm{~T}_{r f}(\mathrm{~K})$ & 19 & 21 & 30 \\
$\mathrm{~T}_{I F}(\mathrm{~K})$ & 3.8 & 3.8 & 3.8 \\
$\mathrm{~T}_{m i x}(\mathrm{~K})^{*}$ & 23.3 & 19.8 & 28.2 \\
$G_{m i x}^{D S}(\mathrm{~dB})$ & -1.6 & -2.3 & -3.2 \\
$G_{r f}(\mathrm{~dB})$ & -0.29 & -0.32 & -0.46 \\
$G_{b s}(\mathrm{~dB})$ & -0.15 & -0.22 & -0.32 \\
$\mathrm{~T}_{m i x} /\left(G_{r f}\right)(\mathrm{K})$ & 25 & 21.3 & 31.3 \\
$\mathrm{~T}_{I F} /\left(G_{r f} G_{m i x}^{D S}\right)(\mathrm{K})$ & 7.5 & 7.0 & 9.0 \\
Measured $\alpha$ & 0.66 & 0.64 & 0.62 \\
\hline \hline
\end{tabular}

LHe stage. For optimal RF bandwidth and performance, we use a corrugated feedhorn ${ }^{11}$ with $\sim 43 \%$ fractional bandwidth. The design is based on numerical simulations of a $180-280-\mathrm{GHz}$ feedhorn with 64 sections by Lamb [41]. Calculated input return loss of the horn is better than $18 \mathrm{~dB}$, the cross-polar component less than $-32 \mathrm{~dB}$, and the phase front error of 0.1 . At $345 \mathrm{GHz}$, the horn has a $f / 2.42$ beam divergence. The optics is designed to provide a $11.8-\mathrm{dB}$ frequency-independent illumination of the telescope's secondary mirror [42]. To check the level of cross-polarization and off-axis aberration of the elliptical mirror (M6) design, a physical optics calculation ${ }^{12}$ was done by Jellema and Finn [43]. In our design, the second focus of the elliptical mirror is positioned at the $77-\mathrm{K}\left(L N_{2}\right)$ stage of the cryostat. This allows the use of a 32-mm-diameter pressure window $\left(7 \omega_{o}\right)$. The IR blocking filters on the 4- and $77-\mathrm{K}$ stages are made of one layer $(100 \mu \mathrm{m}) \mathrm{G} 104$ and one layer $(200 \mu \mathrm{m})$ G108 Zitex (30\%-60\% porous Teflon $\left.{ }^{13}\right)$, separated by $\sim 40 \mu \mathrm{m}$ vacuum via a precision-cut circular high-density polyethylene (HDPE) spacer. This design has a better than $99 \%$ transmission from 280 to $420 \mathrm{GHz}$, while photon scattering leads to a loss of $\approx 98.5 \%$ per sheet in the thermal IR [44].

The vacuum window is made of $715-\mu \mathrm{m}$ HDPE that is antireflection coated with one layer $(150 \mu \mathrm{m}) \mathrm{G} 106$ Zitex on one side, and one layer $(200 \mu \mathrm{m}) \mathrm{G} 108$ on the other. Calculated transmission is better than $98 \%$ across the $280-420-\mathrm{GHz}$ frequency band. As a "glue" to make the porous Teflon stick to the HDPE, we used $50-\mu \mathrm{m}$-thick low-density polyethylene (LDPE). ${ }^{14}$ This material has a melting point just below that of HDPE, and under pressure on a hot plate, the "sandwich" of Zitex-LDPE-HDPE-LDPE-Zitex fuses together. Fourier transform spectrometer (FTS) transmission measurements confirm the excellent transmission properties of these windows. To inject the LO signal, 15 we use a $25-\mu \mathrm{m}$ quasi-optical Mylar beamsplitter with a calculated reflection of $4.9 \%$ at $345 \mathrm{GHz}$ $\left(G_{\mathrm{bs}}\right.$ in Table IV). Standing waves between the telescope secondary mirror and cryostat are minimized by tilting the vacuum window and IR blocks at $5^{\circ}$ angles.

\footnotetext{
${ }^{11}$ Custom Microwave Inc., Longmont, CO.

${ }^{12}$ TICRA Engineering, Copenhagen, Denmark. [Online]. Available: http:// www.ticra.com

${ }^{13}$ Saint-Gobain Performance Plastics Inc., Wayne, NJ.

${ }^{14}$ Carlisle Plastics Company Inc., New Carlisle, OH.

${ }^{15}$ Virginia Diodes Inc., Charlottesville, VA.
}

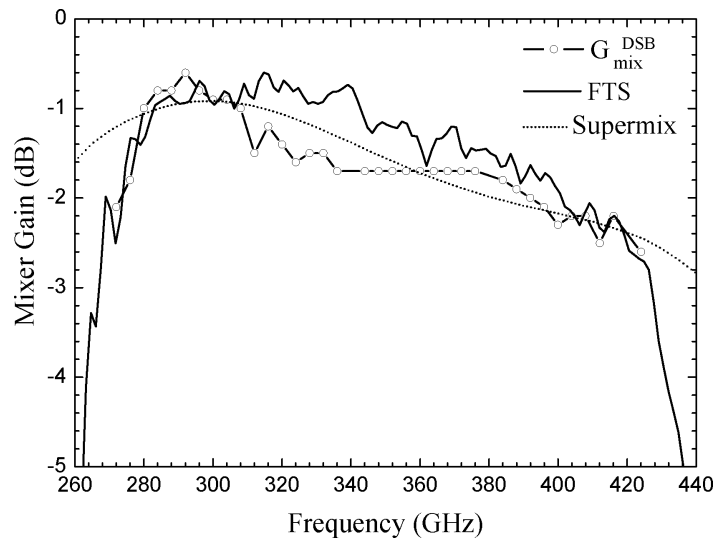

Fig. 9. FTS measurement superimposed with the mixer conversion gain and "Supermix" simulation (no optics/waveguide). The RF bandwidth is limited, $20 \mathrm{GHz}$ on either side, by the frequency response of the corrugated feedhorn. The waveguide cutoff frequency is $250 \mathrm{GHz}$.

\section{RECEIVER PERFORMANCE}

\section{A. FTS Measurements}

To investigate the coupling to the twin SIS junction RF matching network, we have measured the direct-detection response of the mixer with an FTS. The result, overlaid with the derived mixer conversion gain and Supermix simulation, is shown in Fig. 9.

A few points are noteworthy. First, the response bandwidth is limited by the corrugated feedhorn ( $\sim 43 \%$ fractional bandwidth), more or less $20 \mathrm{GHz}$ symmetrically on either side. Second, the direct-detection response is centered on $350 \mathrm{GHz}$. This argues for the accuracy of the computer simulations and quality of the device fabrication.

\section{B. Heterodyne Results and Discussion}

In Fig. 10, we show the measured heterodyne response and associated LO pumped and unpumped $I / V$ curves at 280,345 , and $424 \mathrm{GHz}$. Optimal mixer bias occurs between 2.1-2.2 mV for all frequencies (see also Fig. 11). Optimal LO pump current is $80-85 \mu \mathrm{A}$, which is $55-60 \mu \mathrm{A}$ over the dark current. From this we calculate that $\alpha \equiv e V_{\mathrm{LO}} / \hbar \omega=0.64$ at $345 \mathrm{GHz}$, corresponding to an available LO power at the two junctions of $160 \mathrm{nW}$. The magnetic field required to suppress the Josephson current to a first minima (one flux quantum) results in an unexpectedly good receiver noise temperature at frequencies below $370 \mathrm{GHz}$. This effect is understood to be due to ac-Josephson oscillations mixing with the third harmonic of the LO signal. By softening the gap with a stronger magnetic field (second null), the harmonic conversion efficiency is reduced, alleviating the problem. The generation of significant amounts of harmonic content may be characteristic of high current-density AlN-barrier SIS junctions. Operating the mixer at the second Josephson null reduces the mixer conversion gain by $\sim 1.5 \mathrm{~dB}$, and has a minimal impact on the receiver noise temperature. The input loads $\left(T_{h}, T_{c}\right)$ are defined using the Callen and Welton formalism [46], [47], where the vacuum zero-point fluctuation noise is included in the blackbody radiation temperature. At the frequencies of interest, this approaches the Rayleigh-Jeans limit. 


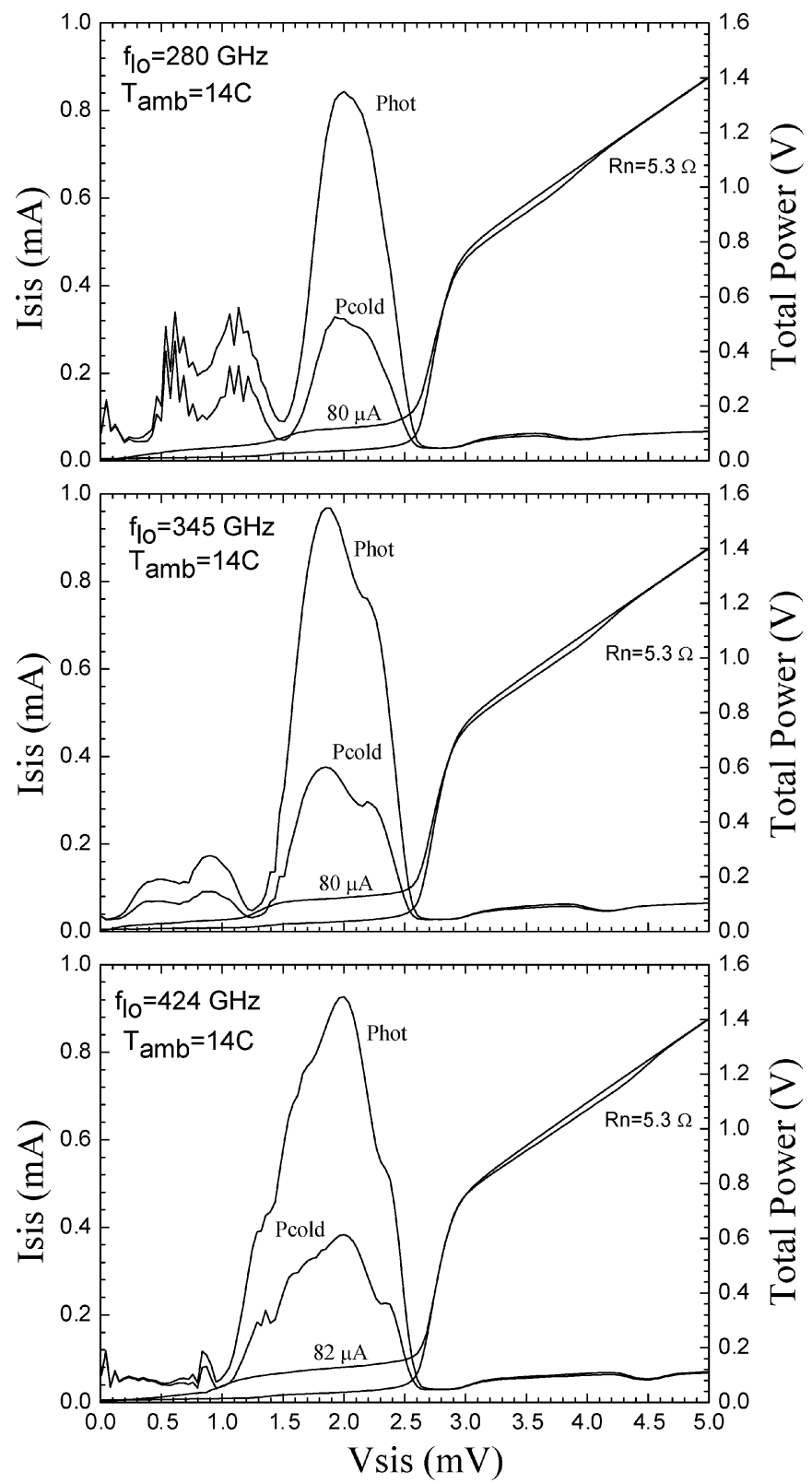

Fig. 10. Measured heterodyne response at 280,345 , and $424 \mathrm{GHz}$ at the CSO (4200 m). Optimal bias ranges from 2.1 to $2.2 \mathrm{mV}$ with an LO pump current of 57-60 $\mu \mathrm{A}$ over the leakage current $(25 \mu \mathrm{A})$. At $345 \mathrm{GHz}$, this corresponds to $\alpha \equiv e V_{\mathrm{LO}} / \hbar \omega=0.64$. The combined twin-junction normal-state resistance $\left(R_{n}\right)$ equals $5.32 \Omega$ and the resistive subgap-to- $R_{n}$ ratio $\simeq 15$.

As a general principle, the receiver should not be biased for maximum $G_{\text {mix }}^{\mathrm{DSB}}$, which occurs when the IF output power is optimized. Rather, the mixer should be biased for optimal sensitivity (Fig. 11). This in effect is similar to "noise matching," as opposed to "power matching" of low noise amplifiers.

To characterize the IF noise contribution, Rudner et al. [48], and Woody et al. [5] proposed to use the unpumped junction above the gap voltage as a calibrated shot noise source [see (5)]. Studies by Dubash et al. [49], [50] quantitatively verified that the noise current of an unpumped SIS junction above the gap is, in fact, the shot noise associated with the direct current. Using this technique, the IF noise contribution and mixer conversion gain were computed as explained by Wengler and Woody [51].

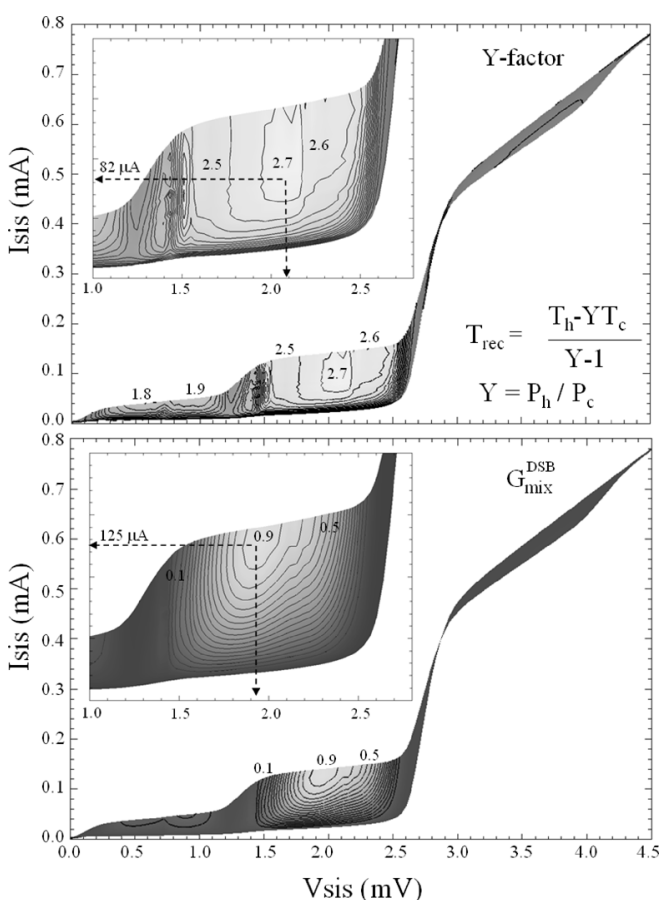

Fig. 11. (top) $Y$-factor (sensitivity) as a function of mixer bias and LO pump current. Optimal sensitivity occurs around $2.1 \mathrm{mV}$ and $82 \mu \mathrm{A}$. (bottom) Conversion gain (linear) versus mixer bias and LO pump current. Maximum conversion gain (IF power) occurs at $1.9-\mathrm{mV}$ bias and $125 \mu \mathrm{A}$ of LO pump current. Contour lines are in steps of 0.1 , and the LO pump frequency is $345 \mathrm{GHz}$. Biased at optimal sensitivity, the mixer gain drops from 0.9 to $0.6(-2.3 \mathrm{~dB})$.

To understand the optics loss in front of the mixer, we employ a technique, commonly known as the "intersecting-line technique," described by Blundell et al. [45] and Ke and Feldman [52]. We find between $280-424 \mathrm{GHz}$ a front-end equivalent noise temperature $\left(T_{\mathrm{rf}}\right)$ of $19-30 \mathrm{~K}$. This includes thermal noise injected via the room-temperature 25- $\mu \mathrm{m}$ Mylar beamsplitter. The uncorrected optical efficiency $\left(G_{\mathrm{rf}}\right)$, referred to $290 \mathrm{~K}$, is found to range from $-0.29 \mathrm{~dB}$ at $280 \mathrm{GHz}$ to $-0.46 \mathrm{~dB}$ at $424 \mathrm{GHz}$. Correcting for the beamsplitter loss $\left(G_{\mathrm{bs}}\right)$, the optical efficiency of the pressure window, IR blocks, and cooled optics is, therefore, $-0.14 \mathrm{~dB}(\sim 96.7 \%)$, which is consistent with the optics design outlined in Section II-G. It should be noted that the "intersecting-line technique" is likely to include some small correction factors because the mixer is not perfectly matched and/or operating in true DSB mode [52]. From fits to our data, the magnitude of this factor is found experimentally to be $\hbar \omega / 2 k$.

The measured receiver noise temperature at $345 \mathrm{GHz}$ is $48 \mathrm{~K}$ DSB. From the shot noise calculations, we obtain at $345 \mathrm{GHz}$ an overall mixer conversion gain of $-2.3 \mathrm{~dB}$, and mixer noise temperature of $19.8 \mathrm{~K}$. Of this, $\sim 9 \mathrm{~K}$ is due to leakage current in the AlN tunnel barrier. The obtained IF noise temperature is $3.8 \mathrm{~K}$ and the IF noise contribution to the receiver budget is $7.0 \mathrm{~K}$. These values are in good agreement with simulation. A detailed breakdown of the noise budget is provided in Table IV. In Fig. 12, we show the heterodyne response from 275 to $425 \mathrm{GHz}$, as measured in the laboratory and at the observatory on top of Mauna Kea, HI. For the balanced heterodyne receivers currently under development, the DSB receiver response is expected to improve in sensitivity by $9-20 \mathrm{~K}$ in the $275-424-\mathrm{GHz}$ 


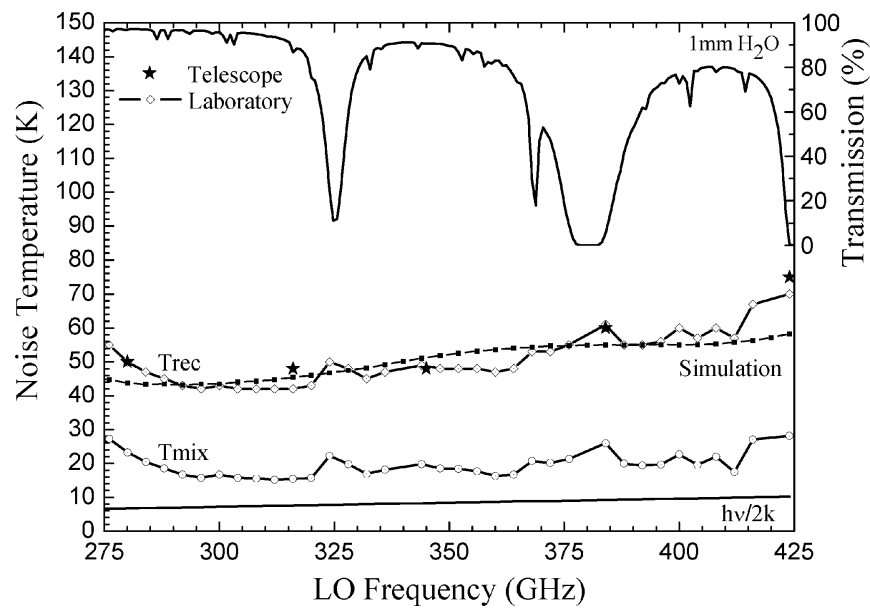

Fig. 12. Measured receiver and mixer noise temperature in the laboratory and at the telescope. The dotted line represents the simulated result. All noise temperatures are uncorrected for optical loss. The atmospheric window for $1 \mathrm{~mm}$ of precipitable water is shown for reference. $T_{\text {mix }}$ includes, due to leakage current below the gap, $9 \mathrm{~K}$ of MAR-enhanced shot noise. In the calculations, the radiometric hot and cold blackbody temperatures are assumed to be in the Rayleigh-Jeans limit.

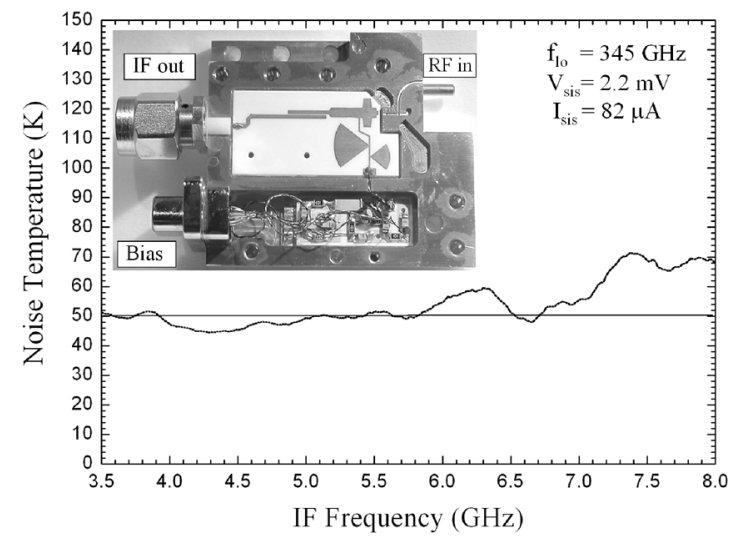

Fig. 13. Receiver noise temperature across the IF band. The rise at $7 \mathrm{GHz}$ is attributed to a slight mistuning of the junction's double-tuned IF response. Computer simulations suggest that this can be corrected with additional wire bonds to ground at the integrated capacitor, and reduction in the IF contact wire-bond length (currently $1 \mathrm{~mm}$ ). The equivalent noise bandwidth of the mixer is $\sim 5 \mathrm{GHz}$.

frequency range due to a reduction in thermal noise and LO amplitude noise.

\section{IF Response}

The 345-GHz IF response of the mixer is shown in Fig. 13. The data was obtained in a $30-\mathrm{MHz}$ resolution bandwidth by scanning a YIG filter ${ }^{16}$ from 3.5 to $8 \mathrm{GHz}$ at $15-\mathrm{MHz}$ intervals. At each frequency, the hot and cold response was measured using an automated chopper wheel. This technique allows efficient integration of the noise in a time frame less than the Allan variance stability time of the instrument $(\sim 9 \mathrm{~s}$ in a $30-\mathrm{MHz}$ IF channel bandwidth). Between 7-8 GHz, we see the noise rise from 50 to $70 \mathrm{~K}$ DSB. Extensive EM field simulations [29], [30] compared to measured IF output data, point to a problem in the assumed "infinite" CPW ground plane and 1-mm-long

\footnotetext{
${ }^{16}$ Micro Lambda Wireless Inc., Fremont, CA.
}

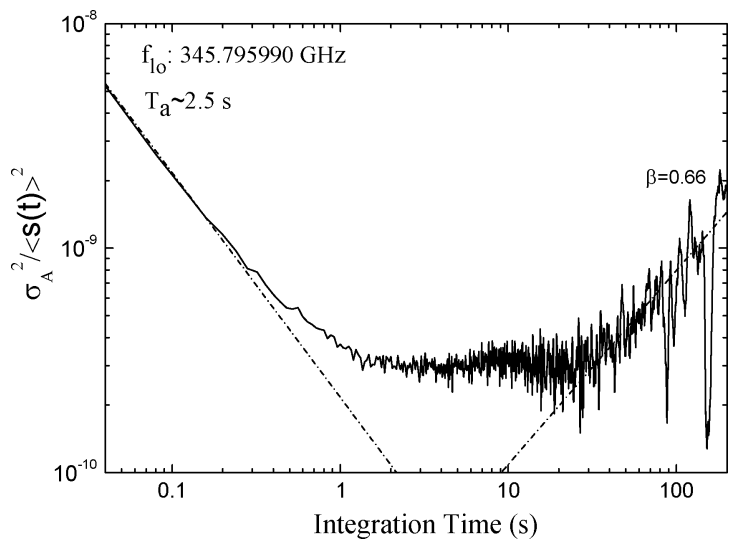

Fig. 14. Measured total-power (continuum) Allan variance on a room-temperature blackbody as a function of integration time with the instrument mounted on the telescope. From the Allan variance analysis, we derive a drift slope of 0.66 and a noise-fluctuation bandwidth $(B)$ of $4.6 \mathrm{GHz}$. At $1 \mathrm{~s}$, a factor of 1.5 is lost in the total-power integration efficiency. For reference, this corresponds to approximately $100 \mathrm{~s}$ in a typical $2-\mathrm{MHz}$ spectrometer channel.

IF bond wire. Since the on-chip IF matching circuit is highly tuned, a slight mistuning in one or more of its components results in a double-peaked passband response. Computer simulations show a mixer IF passband peak at $4 \mathrm{GHz}$, and a $3-\mathrm{dB}$ dip at $8 \mathrm{GHz}$. Fortunately this issue is easily solved with the addition of extra ground contacts in the vicinity of the integrated capacitor (Fig. 3) and a reduction of the IF bond wire length. Due to this effect, the 4-8-GHz receiver noise temperature is slightly weighted toward the 3.5-6-GHz range (Table IV).

\section{Stability}

In general, receiver instabilities lead to a loss in integration efficiency and poor baseline quality [53]. Throughout the design process, attention has been given to the multiplicity of factors that degrade the stability of the instrument. These include improved SIS and LNA bias electronics, voltage-divider networks in the SIS mixer and cryogenic LNAs, enhanced thermal design of the room-temperature IF amplifiers, careful elimination of all ground loops, and the use of twisted-pair wires in the cryostat to minimize microphonic pickup. The resulting Allan variance stability plot is shown in Fig. 14.

It has been found [54], [55] that fluctuations with a $f^{-\alpha}$ power spectrum show up in the Allan variance plot as $T_{\mathrm{int}}^{\alpha-1}$ with $T_{\text {int }}$ defined as the integration time. If we let $\beta=\alpha-1$, the shape of the Allan variance is found to follow

$$
\sigma_{A}^{2}\left(T_{\mathrm{int}}\right)=a T_{\mathrm{int}}^{-1}+b+c T_{\mathrm{int}}^{\beta}
$$

where $a, b$, and $c$ are constants. The first term, with $\beta=-1$, represents radiometric (white) noise. In a $\log -\log$ plot, it has a slope of -1 (Fig. 14). This type of frequency-independent (uncorrelated) noise integrates down with the square root of time according to the well-known radiometer equation [56]

$$
\sigma=\frac{<s(t)>}{\sqrt{B T_{\mathrm{int}}}} .
$$

Here, $s(t)$ is the measured detector IF output signal in the time domain, and $B$ is the equivalent IF noise-fluctuation bandwidth 

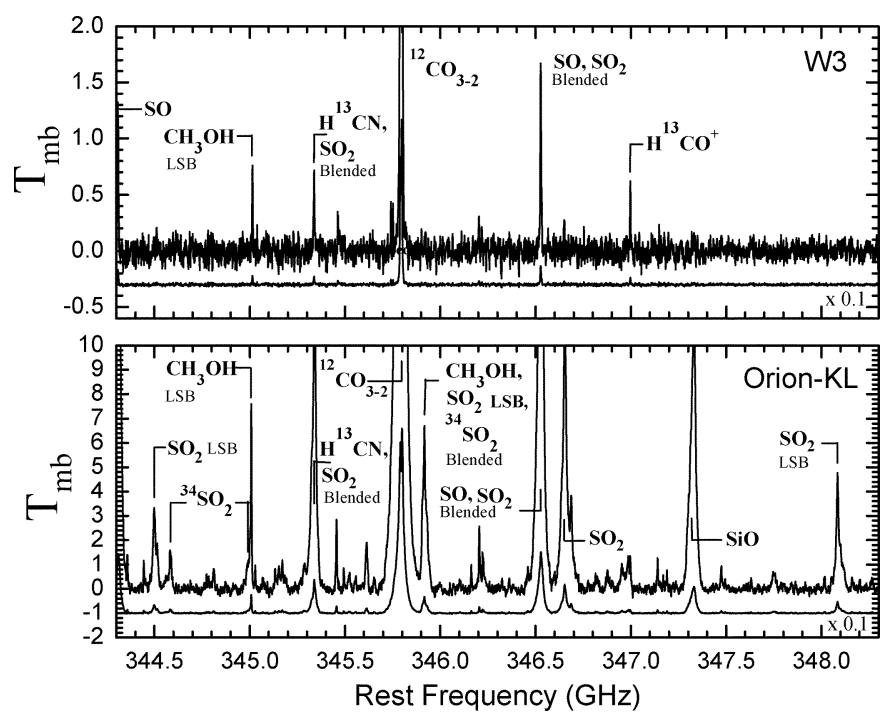

Fig. 15. Test spectra taken at the CSO in October 2006 of the hot core around Orion IRC2 and $\mathrm{W} 3$ in ${ }^{12} \mathrm{CO} J=3 \rightarrow 2$. Due to time constraints and poor weather, pointing was nonoptimal. Line identifications based on Schilke et al. [59] and Helmich and van Dishoeck [60].

of the system. The last term in (7) represents drift noise. In between these two limits, a certain amount of gain fluctuation or flicker noise with a $1 / f$ noise power spectral distribution may exist $(\beta=0)$. The intercept between radiometric and drift noise is the Allan minimum time of the system $\left(T_{A}\right)$. From our measured data, we calculate a total-power Allan minimum time of $2.5 \mathrm{~s}$ and a noise-fluctuation bandwidth of $4.6 \mathrm{GHz}$, consistent with the IF passband shown in Fig. 13.

If the stability were to be limited by drift noise alone, the Allan variance time would scale with IF bandwidth as

$$
T_{A}^{\prime}=T_{A}\left(\frac{B}{B^{\prime}}\right)^{1 /(1+\beta)} .
$$

$T_{A}$ is the measured Allan variance time in a noise-fluctuation bandwidth $B$, and $T_{a}^{\prime}$ is the expected Allan stability time in bandwidth $B^{\prime}$. Note that for optimal observing efficiency, integrations time should be kept well below the Allan minimum stability time of the system. In our case, a 50\% loss in integration efficiency is incurred at $T_{\text {int }}=1 \mathrm{~s}(B=4.6 \mathrm{GHz})$. Using (9), this corresponds to approximately $100 \mathrm{~s}$ in a $2-\mathrm{MHz}$ spectrometer channel noise bandwidth. These results are measured with the instrument mounted on the telescope and are a factor 6-8 times better than the existing facility heterodyne receivers. For comparison, the ALMA [58] specified goal for total-power gain stability $(\partial G / G)$ at $1 \mathrm{~s}$ is $10^{-4}$. The results presented here equate to a normalized total-power gain stability $(\sigma /\langle s(t)\rangle)$ of $1.5 \times 10^{-5}$.

\section{E. Observations}

In October 2006, we observed the ${ }^{12} \mathrm{CO} J=3 \rightarrow 2$ $(345.796 \mathrm{GHz})$ transition in the hot core regions around Orion IRC2 and W3 at the CSO (Fig. 15). The single-sideband (SSB) system temperature, air mass, and integration times were 1380 $\mathrm{K}$ and 1.31 and 8.4 min for the Orion observation, and $1430 \mathrm{~K}$ and 1.45 and $10.1 \mathrm{~min}$ for the $\mathrm{W} 3$ observation. Consistent with our optics design, fits to beam measurements on the telescope's secondary mirror give a Gaussian illumination with 11.5-dB edge taper. From this and knowledge of the primary surface roughness $(20 \mu \mathrm{m})$, we estimate a main-beam efficiency of $\sim 75 \%$. The weather conditions were poor during our engineering run with a $225-\mathrm{GHz}$ zenith atmospheric opacity $\left(\tau_{225}\right)$ $\geq 0.205$. At $345.796 \mathrm{GHz}$, this translates into an air-mass corrected on-source opacity $(\tau)$ of 0.92 for Orion and 1.02 for W3 [57].

The measured SSB system temperatures $\left(T_{\mathrm{sys}}^{\mathrm{SSB}}\right)$ are consistent with those obtained from theory

$$
T_{\mathrm{sys}}^{\mathrm{SSB}}=2\left(\frac{T_{\mathrm{rec}}^{\mathrm{DSB}}+\left(1-\eta_{s} e^{-\tau}\right) T_{\mathrm{sky}}}{\eta_{s} e^{-\tau}}\right) .
$$

$\eta_{s}$, the hot spillover efficiency, is $90 \%$ and was obtained from sky-dip measurements in July 2006. The physical temperature of the sky $\left(T_{\text {sky }}\right)$ is estimated to be $\sim 275 \mathrm{~K}$. Given a $50-\mathrm{K}$ DSB receiver noise temperature (Fig. 12), we obtain a theoretical $T_{\text {sys }}^{\mathrm{SSB}}$ of $1260 \mathrm{~K}$ for Orion-KL and $1450 \mathrm{~K}$ for $\mathrm{W} 3$. This includes a respective SSB atmospheric noise contribution of 825 and $970 \mathrm{~K}$. In the event an SSB receiver with $10-\mathrm{dB}$ sideband rejection ratio (ALMA) and $T_{\mathrm{rec}}^{\mathrm{SSB}}=2 \times T_{\mathrm{rec}}^{\mathrm{DSB}}$ had been used for the observations, the SSB system temperatures are estimated to have been 820 and $940 \mathrm{~K}$. Though much improved over the single-ended DSB receiver (a factor of 2.4 in integration time), the system temperature would still be limited by the sky.

\section{CONCLUSION}

We have discussed the design, development, and installation of a 275-425-GHz tunerless heterodyne receiver. By combining AlN-barrier high-current-density $\left(25 \mathrm{kA} / \mathrm{cm}^{2}\right)$ SIS technology with a full-height waveguide to thin-film microstrip transition, we are able to achieve an unprecedented $43 \%$ instantaneous bandwidth, limited primarily by the mixer corrugated feedhorn.

From 275 to $425 \mathrm{GHz}$, we measure a receiver noise temperature of 40-72 K DSB. In this frequency range, the mixer gain is relatively constant at $-2.3 \pm 1 \mathrm{~dB}$. The optimal mixer bias is found between $2.1-2.2 \mathrm{mV}$, with an LO pumped SIS current of 55-60 $\mu \mathrm{A}$ over the $25-\mu \mathrm{A}$ leakage current at all frequencies. These parameters allow for easy automation of the instrument.

The high current-density AlN-barrier devices are found to have a somewhat higher leakage current then is commonly observed in lower current-density $\mathrm{AlO}_{x}$ tunnel junctions. We find that below the gap, $90 \%$ of the leakage current induced shot noise is via single-electron tunneling, with only $10 \%$ due to MARs. The total MAR enhanced shot noise is $\sim 8.9 \mathrm{~K}$. This accounts for $\sim 45 \%$ of the obtained $19.8-\mathrm{K}$ intrinsic mixer noise temperature.

To optimize integration efficiency and baseline quality, a significant effort has been expended to achieve maximum instrument stability. The measured total-power stability on the telescope is $\sim 2.5 \mathrm{~s}$ in $4.6 \mathrm{GHz}$, or approximately $100 \mathrm{~s}$ in a $2-\mathrm{MHz}$ noise-fluctuation bandwidth. This is a factor 6-8 times better than the existing facility heterodyne instrumentation.

\section{ACKNOWLEDGMENT}

The authors wish to thank J. Lamb, OVRO Millimeter Group, California Institute of Technology, Pasadena, for help with 
modeling the corrugated feedhorn, W. Jellema, Netherlands Institute for Space Research SRON, Groningen, The Netherlands, and T. Finn, National University of Ireland, Maynooth, Ireland, for their physical optics calculations on M6, R. Chamberlin, Caltech Submillimeter Observatory (CSO), Hilo, HI, for his help in setting up the PLL, for CSO manpower allocation, and for test spectra, J. Groseth, California Institute of Technology, for laying out the needed bias electronics PCBs, D. Warden, California Institute of Technology, and P. Nelson, CSO, for their diligence in assembling the many bias boxes and cryostat, A. Guyer, CSO, and E. Bufil, CSO, for help with the mounting hardware, N. Wadefalk and P. Starsky, both with the Chalmers University of Technology, Göteborg, Sweden, for their help and advice with the IF microwave electronics and for supplying the $2.2 \mathrm{~K}$ LNAs, S. Weinreb, Jet Propulsion Laboratory and California Institute of Technology, for making available the cryogenic low noise MMICs, T. Vayonakis, California Institute of Technology, for his help with AR coating of the infrared and pressure windows, F. Rice, California Institute of Technology, for support of Supermix, P. Dieleman, Netherlands Institute for Space Research SRON, for his advice on modeling MAR in our AlN-barrier SIS junctions, and J. Zmuidzinas, California Institute of Technology, for general advise and insight.

\section{REFERENCES}

[1] J. W. Kooi, A. Kovács, S. Kaye, J. Dama, J. Zmuidzinas, and T. G. Phillips, "Heterodyne instrumentation upgrade at the Caltech Submillimeter Observatory," Proc. SPIE, vol. 4855, pp. 265-278, Feb. 2003.

[2] J. W. Kooi, A. Kovács, B. Bumble, G. Chattopadhyay, M. L. Edgar, S. Kaye, R. LeDuc, J. Zmuidzinas, and T. G. Phillips, "Heterodyne instrumentation upgrade at the Caltech Submillimeter Observatory II," Proc. SPIE, vol. 5498, pp. 332-348, Jun. 2004.

[3] C. R. Predmore, N. R. Erickson, G. R. Huguenin, and P. Goldsmith, "A continuous comparison radiometer at $97 \mathrm{GHz}$," IEEE Trans. Microw. Theory Tech., vol. MTT-33, no. 1, p. 1267, Jan. 1985.

[4] D. Maier, A. Barbier, B. Lazareff, and K. F. Schuster, "The ALMA band 7 mixer," in Proc. 16th Int. Space Terahertz Technol. Symp., Göteborg, Sweden, May 2005, Paper S08-02.

[5] D. P. Woody, R. E. Miller, and M. J. Wengler, " $85-115 \mathrm{GHz}$ receivers for radio astronomy," IEEE Trans. Microwave Theory Tech., vol. MTT-33, no. 2, pp. 90-95, Feb. 1985.

[6] B. N. Ellison and R. E. Miller, "A low noise $230 \mathrm{GHz}$ SIS receiver," Int. J. Infrared Millim. Waves, vol. 8, pp. 609-625, Jun. 1987.

[7] J. W. Kooi, M. Chan, T. G. Phillips, B. Bumble, and H. G. Leduc, "A low noise $230 \mathrm{GHz}$ heterodyne receiver employing $0.25 \mu \mathrm{m}^{2}$ area $\mathrm{Nb} / \mathrm{AlO}_{x} / \mathrm{Nb}$ tunnel junctions," IEEE Trans. Microw. Theory Tech., vol. 40, no. 5, pp. 812-815, May 1992.

[8] C. K. Walker, J. W. Kooi, M. Chan, H. G. Leduc, P. L. Schaffer, J. E. Carlstrom, and T. G. Phillips, "A low-noise $492 \mathrm{GHz}$ SIS waveguide receiver," Int. J. Infrared Millim. Waves, vol. 13, pp. 785-798, Jun. 1992.

[9] J. H. Kawamura, T. R. Hunter, C.-Y. E. Tong, R. Blundell, Q. Zhang, C. A. Katz, D. C. Papa, and T. K. Sridharan, "First image with the CfA superconducting HEB receiver: The protostellar outflow from IRAS $20126+4104$ in CO $(J=7-6)$, , Pubs. Astronom. Soc. Pacific, vol. 111, pp. 1088-1094, Sep. 1999.

[10] R. L. Eisenhart and P. J. Khan, "Theoretical and experimental analysis of a waveguide mounting structure," IEEE Trans. Microw. Theory Tech., vol. MTT-19, no. 8, pp. 706-717, Aug. 1971.

[11] S. Withington and G. Yassin, "Analytical expression for the input impedance of a microstrip probe in waveguide," Int. J. Infrared Millim. Waves, vol. 17, pp. 1685-1705, Nov. 1996.

[12] C.-Y. E. Tong, R. Blundell, and S. Paine, "Design and characterization of a $250-350-\mathrm{GHz}$ fixed-tuned superconductor-insulator-superconductor receiver," IEEE Trans. Microw. Theory Tech., vol. 44, no. 9 , pp. 1548-1556, Sep. 1996.
[13] Y. Delorme, M. Salez, B. Lecomte, I. Péron, F. Dauplay, A. Féret, J. Spatazza, J. -M. Krieg, and K. F. Schuster, "Space-qualified SIS mixers for Herschel Space Observatory's HIFI band 1 instrument," in Proc. 16th Int. Space Terahertz Technol. Symp., Göteborg, Sweden, May 2005, Paper S08-05.

[14] C. F. J. Lodewijk, M. Kroug, and T. M. Klapwijk, "Improved design for low noise Nb SIS devices for band 9 of ALMA (600-720 GHz)," in Proc. 16th Int. Space Terahertz Technol. Symp., Göteborg, Sweden, May 2005, Paper S03-05.

[15] B. D. Jackson, G. de Lange, T. Zijlstra, M. Kroug, J. W. Kooi, J. A. Stern, and T. M. Klapwijk, "Low-noise 0.8-0.96- and 0.96-1.12 THz superconductor-insulator-superconductor mixers for the Herschel Space Observatory," IEEE Trans. Microw. Theory Tech., vol. MTT-54, no. 2, pp. 547-558, Feb. 2006.

[16] Y.-C. Leong and S. Weinreb, "Full-band waveguide-to-microstrip probe transitions," in IEEE MTT-S Int. Microw. Symp. Dig., Anaheim, CA, Jun. 13-19, 1999, pp. 1435-1438.

[17] J. H. C. van Heuven, "A new integrated waveguide-microstrip transition," IEEE Trans. Microw. Theory Tech., vol. MTT-24, no. 3, pp. 144-147, Mar. 1976.

[18] A. R. Kerr and S. K. Pan, "Some recent developments in the design of SIS mixers," Int. J. Infrared Millim. Waves, vol. 11, no. 10, pp. 1169-1187, Nov. 1990.

[19] A. R. Kerr, "Elements for E-plane split-block waveguide circuits," NRAO, Charlottesville, VA. [Online]. Available: http://www.alma. nrao.edu/memos/html-memos/alma381/memo381.pdf

[20] S. -K. Pan, A. R. Kerr, M. W. Pospiezalski, E. F. Lauria, W. K. Crady, N. Horner, Jr., S. Srikanth, E. Bryerton, K. Saini, S. M. X. Claude, C. C. Chin, P. Dindo, G. Rodrigues, D. Derdall, J. Z. Zhang, and A. W. Lichtenberger, "A fixed-tuned SIS mixer with ultra-wide-band if and quantum-limited sensitivity for ALMA band 3 (84-116 GHz) receivers," in Proc. 15th Int. Space Terahertz Technol. Symp., Northampton, MA, Apr. 2004, pp. 62-69.

[21] S. Withington, G. Yassin, J. Leech, and K. G. Isaak, "An accurate expression for the input impedance of one-sided microstrip probes in waveguide," in Proc. 10th Int. Space Terahertz Technol. Symp., Charlottesville, VA, Mar. 1999, pp. 508-518.

[22] J. W. Kooi, G. Chattopadhyay, S. Withington, F. Rice, J. Zmuidzinas, C. K. Walker, and G. Yassin, "A full-height waveguide to thin-film microstrip transition with exceptional RF bandwidth and coupling efficiency," Int J. Infrared Millim. Waves, vol. 24, no. 3, pp. 261-284, Sep. 2003.

[23] A. Navarrini, B. Lazareff, D. Billon-Pierron, and I. Peron, "Design and characterization of 225-370 GHz DSB and 250-360 GHz SSB full height waveguide SIS mixers," in Proc. 13th Int. Space Terahertz Technol. Symp., Cambridge, MA, Mar. 2002, pp. 33-40.

[24] C. Risacher, V. Vassilev, A. Pavolotsky, and V. Belitsky, "Waveguide-to-microstrip transition with integrated bias-T," IEEE Microw. Wireless Compon. Lett., vol. 13, no. 7, pp. 262-264, Jul. 2003.

[25] C. Risacher, V. Belitsky, V. Vassilev, I. Lapkin, and A. Pavolotsky, "A 275-370 GHz SIS receiver with novel probe structure," Int. J. Infrared Millim. Waves, vol. 26, no. 6, pp. 867-880, May 2005

[26] F. P. Mena, J. W. Kooi, A. M. Baryshec, D. F. J. Ledewaijk, R. Hesper, and W. Wild, "Construction of a sideband separating heterodyne mixer for band 9 of ALMA," in Proc. 17th Int. Space Terahertz Technol. Symp., Pasadena, Ca., Mar. 2007, to be published.

[27] J. Ward, F. Rice, and J. Zmuidzinas, "Supermix: A Flexible software library for high-frequency circuit simulation, including SIS mixers and superconducting components," in Proc. 10th Int. Symposium on Space Terahertz Technology, Charlottesville, VA, Mar. 1999, pp. 269-281.

[28] F. Rice, M. Sumner, J. Zmuidzinas, R. Hu, H. LeDuc, A. Harris, and D. Miller, "SIS mixer design for a broadband millimeter spectrometer suitable for rapid line surveys and redshift determinations," in Proc. SPIE, Kona, HI, 2002, vol. 4855, pp. 301-311.

[29] HFSS. Ansoft Corporation, Pittsburgh, PA, 2005.

[30] SONNET. Sonnet Software, Liverpool, NY, 2005.

[31] J. W. Kooi, M. Chan, B. Bumble, H. G. LeDuc, P. L. Schaffer, and T. G. Phillips, "180-425 GHz low noise SIS waveguide receivers employing tuned $\mathrm{Nb} / \mathrm{AlO}_{x} / \mathrm{Nb}$ tunnel junctions," Int. J. Infrared Millim. Waves, vol. 15, no. 5, pp. 783-805, May 2004.

[32] D. Maier, S. Devoluy, M. Schicke, and K. F. Schuster, " 230 GHz SSB SIS mixer for band 3 of the new generation receivers for the Plateau de Bure interferometer," in Proc. 16th Int. Space Terahertz Technol. Symp., Göteborg, Sweden, May 2005, Paper S03-02.

[33] A. R. Kerr, "Saturation by noise and CW signals in SIS mixers," in Proc. 13th Int. Space Terahertz Technol. Symp., Cambridge, MA, Mar. 2002, pp. 11-22. 
[34] P. Dieleman, H. G. Bukkems, T. M. Klapwijk, M. Schicke, and K. H. Gundlach, "Observation of Andreev reflection enhanced shot noise," Phys. Rev. Lett., vol. 79, pp. 3486-3489, Nov. 1997.

[35] W. Menzel, L. Zhu, K. Wu, and F. Bögelsack, "On the design of novel compact broadband planar filters," IEEE Trans. Microw. Theory Tech., vol. 51, no. 2, pp. 364-370, Feb. 2003.

[36] G. de Lange, SRON, NL, private communication, 2003.

[37] H. Golstein, SRON, NL, private communication, 2003.

[38] N. Wadefalk, A. Mellberg, I. Angelov, M. Barsky, S. Bui, E. Choumas, R. Grundbacher, E. Kollberg, R. Lai, N. Rorsman, P. Starski, J. Stenarson, D. Streit, and H. Zirath, "Cryogenic, wide-band, ultra-lownoise IF amplifiers operating at ultra-low DC-power," IEEE Trans. Microw. Theory Tech., vol. 51, no. 6, pp. 1705-1711, Jun. 2003.

[39] N. Wadefalk, private communication, California Inst. Technol., Pasadena, CA, 2005.

[40] N. Wadefalk and S. Weinreb, "Very low-noise amplifiers for very large arrays," in IEEE MTT-S Int. Microw. Symp. Dig., Long Beach, CA, Jun. 2005.

[41] J. Lamb, private communication, California Inst. Technol., Pasadena, CA, 2003.

[42] P. F. Goldsmith, Quasioptical Systems. Piscataway, NJ: IEEE Press, 1998.

[43] W. Jellema and T. Finn, SRON, NL, private communication, 2005.

[44] D. J. Benford, M. C. Gaidis, and J. W. Kooi, "Transmission properties of ZITEX in the infrared to submillimeter," in Proc. 10th Int. Space Terahertz Technol. . Symp, Mar. 1999, pp. 402-411.

[45] R. Blundell, R. E. Miller, and K. H. Gundlach, "Understanding noise in SIS receivers," Int. J. Infrared Millim. Waves, vol. 13, no. 1, pp. 3-26, Jan. 1992.

[46] H. B. Callen and T. A. Welton, "Irreversibility and generalized noise," Phys. Rev., vol. 83, no. 1, pp. 34-40, Jul. 1951.

[47] A. R. Kerr, M. J. Feldman, and S. K. Pan, "Receiver noise temperature, the quantum noise limit, and the role of the zero-point fluctuations," Electron. Div., NRAO , Charlottesville, VA, Internal Rep. 304, Sep. 1996.

[48] S. Rudner, M. J. Feldman, E. Kollberg, and T. Claeson, "Superconducting-insulator-superconducting mixing with arrays at millimeterwave frequencies," J. Appl. Phys., vol. 52, pp. 6366-6371, 1981.

[49] N. B. Dubash, G. Pance, and M. J. Wengler, "Photon noise in the SIS detector," IEEE Trans. Microw. Theory Tech., vol. 42, no. 4, pp. 716-725, Apr. 1994.

[50] N. B. Dubash, M. J. Wengler, and J. Zmuidzinas, "Shot noise and photon-induced correlations in $500 \mathrm{GHz}$ SIS detectors," IEEE Trans. Appl. Supercond., vol. 5, no. 2, pp. 3308-3311, Jun. 1995.

[51] M. J. Wengler and D. P. Woody, "Quantum noise in heterodyne detection,” IEEE J. Quantum Electron., vol. QE-23, no. 5, pp. 613-622, May 1987.

[52] Q. Ke and M. J. Feldman, "A technique for noise measurements of SIS receivers," IEEE Trans. Microw. Theory Tech., vol. 42, no. 4, pp. 752-755, Apr. 1994.

[53] J. W. Kooi, G. Chattopadhyay, M. Thielman, T. G. Phillips, and R. Schieder, "Noise stability of SIS receivers," Int J. Infrared Millim. Waves, vol. 21, no. 5, pp. 689-716, May 2000.

[54] R. Schieder and C. Kramer, "Optimization of heterodyne observations using Allan variance measurements," Astron. Astrophys. 373, pp. 746-756, Jul. 2001.

[55] V. Ossenkopf, "A unified Allan variance computation scheme," Physik. Insitut, Univ. Köln, Cologne, Germany, Jul. 2003. [Online]. Available: http://www.ph1.uni-koeln.de/ftpspace/users/ossk/preprints

[56] J. D. Kraus, Radio Astronomy. New York: McGraw-Hill, 1966, ch. 3 and 7.

[57] J. R. Pardo, J. Cernicharo, and E. Serabyn, "Atmospheric transmission at microwaves (ATM): An improved model for $\mathrm{mm} / \mathrm{submm}$ applications," IEEE Trans. Antennas Propag., vol. 49, no. 12, pp. 1683-1694, Dec. 2001.

[58] M. A. Holdaway, "effects of atmospheric emission fluctuations and gain fluctuations on continuum total power observations with ALMA," NRAO, Charlottesville, VA, ALMA Memo 490, Mar. 30, 2004.

[59] P. Schilke, T. D. Groesbeck, G. A. Blake, and T. G. Phillips, "A line survey of Orion KL from 325 to $360 \mathrm{GHz}$," Astrophys. J., ser. 108, pp. 301-337, Jan. 1997, suppl.

[60] F. P. Helmich and E. F. van Dishoeck, "Physical and chemical variations within the W3 star-forming region, II. The $345 \mathrm{GHz}$ spectral line survey," Astrophys. J., ser. 124, pp. 205-253, Aug. 1997, suppl.
Attila Kovács, photograph and biography not available at time of publication.

Matthew. C. Sumner received the A.B. degree in physics and astronomy from Harvard University, Cambridge, MA, in 1996, and is currently working toward the Ph.D. degree in physics from the California Institute of Technology, Pasadena. His doctoral dissertation will make use of the unique capabilities of the new generation of wideband submillimeter receivers to perform unbiased surveys of molecular-line emission from low-mass protostars.

$\mathrm{He}$ is currently with the California Institute of Technology, where he works on submillimeter astronomy and instrumentation. He has been involved with the installation, testing, and use of two prototype receivers at the Caltech Submillimeter Observatory (CSO), Mauna Kea, HI.

Goutam Chattopadhyay (S'93-M'99-SM'01) received the B.E. degree in electronics and telecommunication engineering from the Bengal Engineering College, Calcutta University, Calcutta, India, in 1987, the M.S. degree in electrical engineering from the University of Virginia, Charlottesville, in 1994, and the Ph.D. degree in electrical engineering from the California Institute of Technology, Pasadena, in 1999.

From 1987 to 1992, he was a Design Engineering with the Tata Institute of Fundamental Research (TIFR), Pune, India, where he designed LO systems for the Giant Meterwave Radio Telescope (GMRT) project. In January 1993, he joined the University of Virginia. In September 1994, he joined the California Institute of Technology, Pasadena. He is currently a Senior Member of the engineering staff of the Jet Propulsion Laboratory (JPL), California Institute of Technology, and a Visiting Faculty with the Department of Physics, California Institute of Technology. His research interests include microwave, millimeter- and submillimeter-wave heterodyne and direct detector receivers, frequency sources and mixers at terahertz frequencies, antennas, SIS mixer technology, direct detector bolometer instruments, and high-frequency radars.

Dr. Chattopadhyay is member of the IEEE Microwave Theory and Techniques Society (IEEE MTT-S) and Eta Kappa Nu. He was the recipient of the 1987 Best Undergraduate Gold Medal presented by the University of Calcutta, the 1992 Jawaharlal Nehru Fellowship Award presented by the Government of India, the 1997 IEEE MTT-S Graduate Fellowship Award, the Award of Excellence from the Jet Propulsion Laboratory (2001, 2003, 2005), and six NASA invention Awards presented by the National Aeronautics and Space Administration (NASA) (2005-2007)

Riley Ceria, photograph and biography not available at time of publication.

Dave Miller, photograph and biography not available at time of publication.

Bruce Bumble, photograph and biography not available at time of publication.

Henry G. LeDuc, photograph and biography not available at time of publication.

Jeffrey A. Stern received the B.S. degree in physics from the Rensselaer Polytechnic Institute, Troy, NY, in 1983, and the Ph.D. degree in applied physics from the California Institute of Technology, Pasadena, in 1991.

$\mathrm{He}$ is currently a Senior Member of the Technical Staff with the Jet Propulsion Laboratory (JPL), Pasadena, CA. His research has focused on a number of superconducting sensors, which includes fabricating superconducting heterodyne mixer chips for the $1200-\mathrm{GHz}$ band of the HIFI instrument on the Herschel Space Observatory. He has also supplied superconducting mixers chips for a number of ground-based observatories for frequencies ranging from 200 to $1400 \mathrm{GHz}$. Most recently, he has focused on fabricating HEB-based mixers using $\mathrm{NbN}$ and $\mathrm{NbTiN}$ and on high-speed single photon detectors using these same materials. 